\title{
A Brief Study Examining the Variability of PRP with Different Preparation Systems
}

\author{
Paul T. Rose, MD, JD, FISHRS I Coral Gables, Florida, USA I paultrose@yahoo.com;
}

Aron G. Nusbaum, MD II Coral Gables, Florida, USA

\begin{abstract}
The use of platelet-rich plasma (PRP) as a means to improve hair growth and limit hair loss has been advocated by numerous hair restoration doctors. Many clinicians and investigators feel that some of the various growth factors attached to platelets can promote hair growth and reactivate senescent hair follicles. Based on various reports in the scientific literature, the response to this type of treatment has been inconsistent at times.

While clinicians use the acronym PRP for all versions of concentrated platelets, it may be that not all PRP preparations are equal. It is conceivable that some of this variation in response may relate to differences in the concentration of platelets produced depending on the PRP system being employed.

In this brief study, the authors evaluated the concentration of platelets produced by four different commercially available systems. The platelet counts in the PRP were compared to whole blood concentration of platelets in each patient. The results demonstrated wide variance in platelet concentrations among the different systems.
\end{abstract}

\section{INTRODUCTION}

The use of PRP for wound healing has been well documented. It has been used for problems ranging from orthopedic injuries to dentistry. In 2005, Uebel reported on the use of PRP as a treatment for hair loss. ${ }^{1,2}$ Other physicians elected to try this therapy for androgenetic alopecia, as well as other types of alopecias, with varying success. ${ }^{3-7}$ Recently, Puig et al. used PRP for the treatment of androgenetic alopecia in women. ${ }^{8}$ The results of that study did not show significant improvement in hair growth.

At the same time, other physicians reported success for male and female pattern androgenetic alopecia. Such variation of response prompted an interest in trying to ascertain what parameters might account for the differences in results. Such parameters include injection method, types of cells included in the preparation, protocol for injection (i.e., frequency of injection, amount injected at each site, micro-needling, or injection directly into the scalp) as well as other factors.

One obvious factor is the concentration of platelets achieved with the system used to create the PRP. In this brief report, the authors analyze the concentration of platelets obtained with four different PRP preparation systems.

\section{MATERIALS AND METHODS}

Four systems for obtaining PRP were evaluated: Arthrex ACP ${ }^{\circledR}$ Double Syringe System (Arthrex Inc.; Naples, Florida), Eclipse PRP ${ }^{\circledR}$ System (Eclipse Aesthetics LLC; The Colony, Texas), Selphyl ${ }^{\circledR}$ System (Factor Medical LLC, Langhorne,
Pennsylvania), and generic yellow-top tubes that were spun to provide platelets.

For each system, four or five patients provided whole blood. The blood was centrifuged and prepared for PRP as instructed per each manufacturer's system and its proprietary centrifuge. Once the PRP was prepared, approximately 1cc of the highest concentrated PRP from the lower part of the plasma was put into an appropriate tube and sent for evaluation at the same independent lab. A Coulter counter was used to provide the platelet concentration.

\section{RESULTS}

The platelet count results are listed in the table below. Looking at the table, you'll note that marked differences were observed in the systems tested. The Eclipse System averaged 3.23 times the whole blood concentration of platelets. The Selphyl System averaged 1.16 times the whole blood concentration of platelets. PRP made from blood placed in a yellow top tube averaged 1.67 times the initial concentration. The Arthrex System gave concentrations that averaged 6.34 times the starting platelet concentration.

$\begin{array}{lrr}\text { PRP Prep } & \text { Whole Blood } & \text { PRP Conc. } \\ \text { Eclipse } & 238 & 298 \\ \text { Eclipse } & 356 & 789 \\ \text { Eclipse } & 243 & 1,548 \\ \text { Eclipse } & 169 & 524 \\ & & \\ \text { Selphyl } & 215 & 309 \\ \text { Selphyl } & 213 & 288 \\ \text { Selphyl } & 222 & 238 \\ \text { Selphyl } & 133 & 190 \\ \text { Selphyl } & 219 & 119 \\ & & \\ \text { Yellow } & 230 & 307 \\ \text { Yellow } & 265 & 419 \\ \text { Yellow } & 186 & 391 \\ & & \\ \text { Arthrex } & 266 & 1,781 \\ \text { Arthrex } & 262 & 1,171 \\ \text { Arthrex } & 428 & 2,053 \\ \text { Arthrex } & 239 & 2,128 \\ \text { Arthrex } & 181 & 1,252\end{array}$

\section{DISCUSSION}

The concentration of the PRP obtained from different commercial systems can vary markedly. Some systems do not seem to concentrate the platelets to any significant extent.

A major difference between the systems is the amount of 
collected whole blood. While most systems require around $15-22 \mathrm{cc}$ of blood, the Arthrex System uses over $120 \mathrm{ml}$ of blood. This typically yields more PRP and may also have led to a higher concentrated, less diluted sample.

It is important to note that one could cite several concerns in such a study. Obviously, the sample size is quite small. The author suggests that others undertake similar studies to demonstrate concentrations achieved with their chosen system(s) and not rely on manufacturers' claims.

It is possible that the samples provided were not optimal for testing with the Coulter counter used. Although the lab did not note clumping, it is possible that clumping of platelets could account for lower concentrations of measured platelets. One might expect such clumping to occur with the other preparations as well if that were the case.

In spite of showing that some systems concentrate platelets to a greater degree than others in this study, one could ask whether platelet concentration is a key determining factor in efficacy for treatment. There is the question of how platelet concentration may relate to growth factor concentration, release, and action etc. Other questions to be answered are, what is the optimal concentration of platelets and is there an inhibitory concentration of platelets.

As for treatment response, we have noted increasing successes with PRP treatment for androgenetic alopecia. Nevertheless, it is unclear as to how often patients should be injected, how much should be injected, or even how PRP should be injected. Some physicians have advocated the use of ACell's MatriStem ${ }^{\circledR}$ (ACell, Inc.; Columbia, Maryland) to enhance the effect of PRP. We have used it with PRP and have observed a positive response.

As a final word of caution, some clinics are preparing PRP by simply spinning down the blood in a laboratory tube used for in vitro testing. Clinicians should be aware that such tubes are not designed for in vivo usage and the FDA has indicated that use of such tubes is illegal. The tubes often have substances that could be dangerous to a patient if injected.

In future studies, it would be helpful for investigators to discuss the important parameters for harvesting PRP and injection techniques so that we are better able to compare systems and results. When comparing systems, techniques, and results, it may be helpful to measure the actual platelet concentration and not rely on the manufacturers' claims.

\section{References}

1. Uebel, C.O. A new advance in baldness surgery using platelet-derived growth factor. Hair Transplant Forum Int'l. 2005; 15(3):77-84.

2. Uebel, C.O., et al. The role of platelet plasma growth factors in male pattern baldness surgery. PRS. 2006; 118(6):1458-1466.

3. Greco, J., and R. Brandt. Preliminary experience and extended applications for the use of autologous platelet-rich plasma in hair transplantation surgery. Hair Transplant Forum Int'l. 2007; 17(4):131-132.

4. Schiavone, G., D. Raskovic, and J. Greco. Platelet rich plasma for androgenetic alopecia: a pilot study. J Dermatol Surg. 2014; 40(9):1010-1019.

5. Gentile, P., et al. The effect of platelet-rich plasma in hair regrowth: a randomized placebo controlled trial. Stem Cells Transl Med. 2015; 4:1317-1323.

6. Li, Z.J., et al. Autologous PR: a potential therapeutic tool for promoting hair growth. J Dermato/ Surg. 2012(Jul); 38(7):1040-1046.

7. Singh, B., and L.J. Goldberg. Autologous platelet-rich plasma for the treatment of pattern hair loss. Am J Clin Dermatol. 2016; 17(4):359-367.

8. Puig, C.J., R. Reese, and M. Peters. Double-blind, placebo-controlled pilot study on the use of platelet-rich plasma in women with female androgenetic alopecia. J Dermatol Surg. 2016; 42(11):1243-1247.

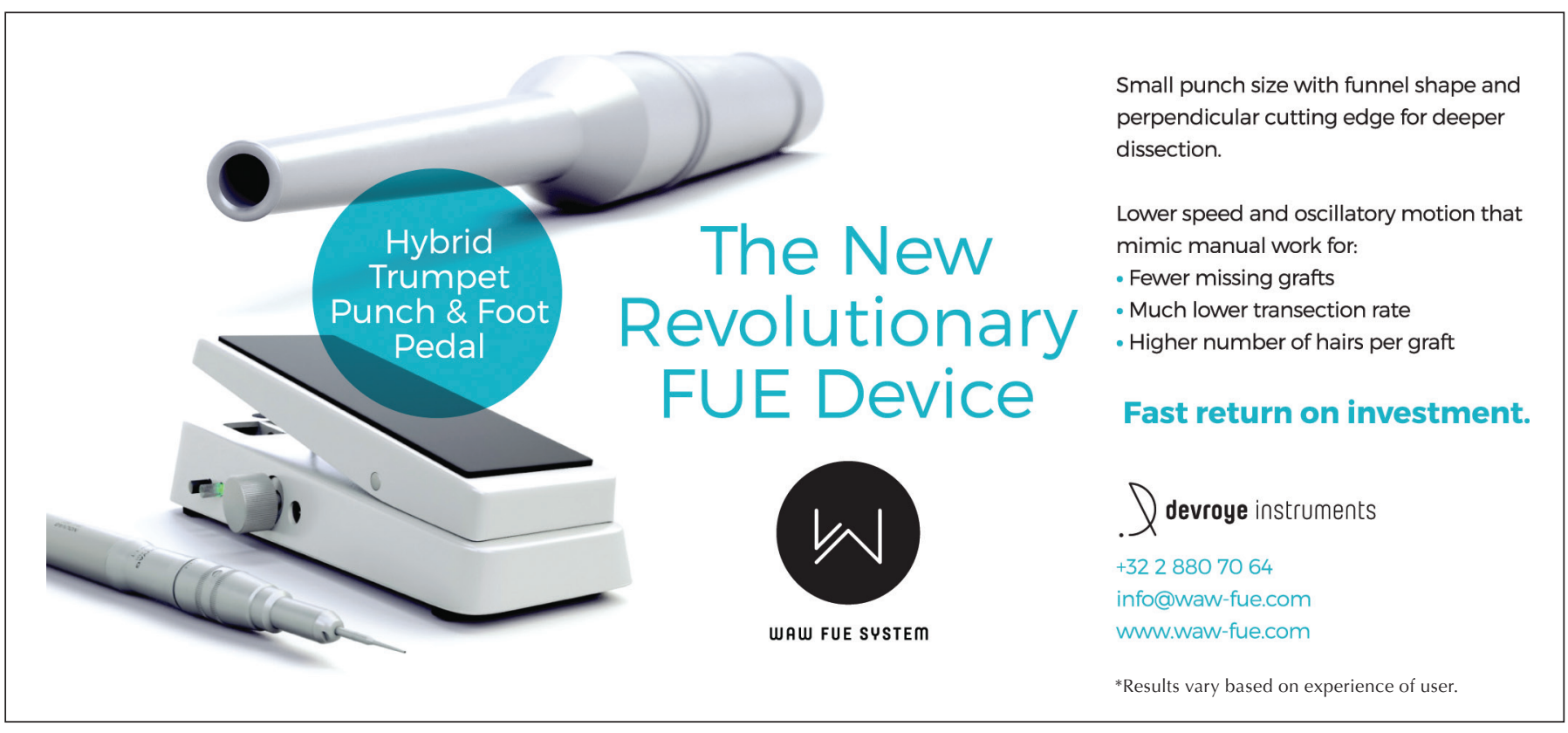

